\title{
On the Spatio-temporal Structure in the Stochastic Diffusive SI Model
}

\author{
Masaaki Ishikawa \\ Graduate School of Science and Engineering, Yamaguchi University \\ 2-16-1 Tokiwadai, Ube 755-8611 Japan \\ E-mail: ishi@yamaguchi-u.ac.jp
}

\begin{abstract}
In this paper, a stochastic diffusive infectious model in the population consisting of the susceptible and the infective is proposed. We consider the proliferation with the strong Allee effect, which means that there exists the optimal population density maximizes the per capita proliferation rate and it becomes negative at the low population density. By numerical simulations, we show that spatio-temporal patterns of the epidemic spreading process become the fractal structure like the Sierpinski gasket in some restricted parameter range and the patterns under the noise are very different from ones in the no noise case in the other restricted parameter range.
\end{abstract}

\section{Introduction}

Recently, many researches on infectious diseases in populations have been done from the mathematical and biological aspects using deterministic and stochastic models [1]-[4]. Many analytical models in epidemiology have been proposed in the past, including SI(susceptible-infected), SIR(susceptible-infectedrecovered) and SIRV(susceptible-infected-recoveredvaccinated) models. Such models in epidemiology can be classified into two types: one is an epidemic model which describes rapid outbreak of infectious diseases in less than one year, and the other is an endemic model which is used in modeling of diseases with longer epidemic period. In this paper, the endemic model with spatial movement in populations consists of the susceptible and the infective, a diffusive SI model, is proposed.

Since the endemic model requires consideration of birth and death in populations, letting densities of the susceptible and the infective at time $t \in \Theta=(0, T)$ and position $x \in G$ be $u(t, x)$ and $v(t, x)$, consider the diffusive SI model:

$$
\begin{gathered}
\frac{\partial u(t, x)}{\partial t}=d_{u} \Delta u(t, x)+f(u(t, x))-b u(t, x) v(t, x), \\
\frac{\partial v(t, x)}{\partial t}=d_{v} \Delta v(t, x)+b u(t, x) v(t, x)-\mu v(t, x),
\end{gathered}
$$

where $\Delta(\cdot)$ is laplacian, the 1st terms of the right-hand sides of Eqs. (1) and (2) denote the change of densities of the susceptible and the infective due to the spatial movement, the 2nd and the 3rd terms are the change of the susceptible density by proliferation and infection, and infection and death of the infective and $b, \mu$ are their infection rate and death rate.

As the function form of the proliferation $f(u)$, the logistic form and proliferation with the Allee effect have been proposed in the past. The Allee effect means that there exists the optimal population density maximizes the per capita proliferation rate defined by $f(u) / u$ as shown in Figs. 1 (a) and 1(b).

In this paper, we adopt the proliferation below:

$$
f(u)=\alpha u(u-\beta)(1-u),
$$

where $\alpha$ is a positive constant and $\beta$ is a constant.

In Eq.(3), the parameter $\beta$ quantifies the intensity of the Allee effect so that it is called "strong" if $0<\beta<1$ and "weak" if $-1<\beta \leq 0$. As shown in Figs. 1(a) and $1(\mathrm{c})$, in the strong Allee effect, the per capita proliferation rate becomes negative for $0<u<\beta$ and when $\beta \leq-1$, the Allee effect is absent. The vertical and horizontal axes in Fig. 1 are the per capita proliferation rate $f(u) / u$ and the density of the susceptible respectively.

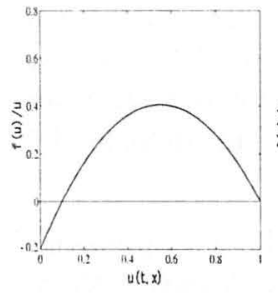

(a) strong

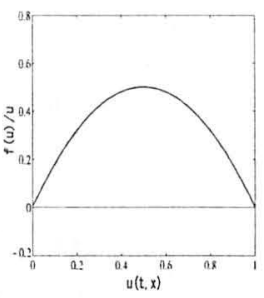

(b) weak

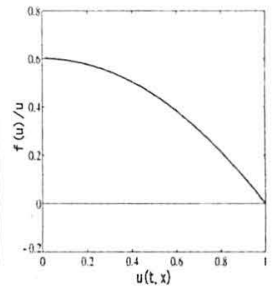

(c) no Allee effect
Fig. 1: Allee effect

We consider the strong Allee effect and the case where the coefficient $\alpha$ related with the proliferation rate contains the random fluctuation and study the influence of the Allee effect and the random fluctuation in proliferation on the spatio-temporal patterns of the infectious disease. 


\section{Stochastic Diffusive SI Model}

Modeling the randomness contained in the parameter $\alpha$ by the spatio-temporal Gaussian white noise $\eta(t, x)$, we replace $\alpha$ so as for the Allee effect to have a meaning by

$$
\alpha \rightarrow(\alpha+\eta(t, x))^{+},
$$

where $f^{+}=\max (f, 0)$ and $\eta(t, x)$ is the spatio-temporal Gaussian white noise with the mean and the covariance such that

$$
\begin{gathered}
E\{\eta(t, x)\}=0 \\
E\left\{\eta\left(t_{1}, x_{1}\right) \eta\left(t_{2}, x_{2}\right)\right\}=K \delta\left(t_{1}-t_{2}\right) \delta\left(x_{1}-x_{2}\right),
\end{gathered}
$$

and where $K$ is the positive constant.

Using Eqs. (3) and (4) to Eq. (1), stochastic diffusive SI model is derived in such a way that

$$
\begin{array}{r}
\frac{\partial u(t, x)}{\partial t}=d_{u} \Delta u(t, x)-b u(t, x) v(t, x) \\
+(\alpha+\eta(t, x))^{+} u(t, x)(u(t, x)-\beta)(1-u(t, x)), \\
\quad(t, x) \in \Theta \times G \\
\frac{\partial v(t, x)}{\partial t}=d_{v} \Delta v(t, x)+b u(t, x) v(t, x)-\mu v(t, x), \quad \\
(t, x) \in \Theta \times G .
\end{array}
$$

The initial and the boundary conditions are given by

$$
u(0, x)=u_{0}(x), \quad v(0, x)=v_{0}(x), \quad x \in G
$$

and

$$
\frac{\partial u(t, x)}{\partial n}=\frac{\partial v(t, x)}{\partial n}=0, \quad(t, x) \in \Theta \times \partial G,
$$

where $\partial G$ is the boundary of $G$ and $\partial(\cdot) / \partial n$ denotes the exterior normal derivative at the boundary $\partial G$.

The random fluctuation in Eq. (7) is considered as the fluctuation caused by the change of environmental situation such as change of weather and the parameter $K$ in Eq. (6) corresponds to the degree of the change of the environmental situation.

\section{Simulations}

In this section, using the stochastic diffusive SI model (7) to (10), we study the influence of the Allee effect, the mortality $\mu$ of the infective and the random fluctuation in the proliferation rate on the spatio-temporal behaviors of the infective by the numerical simulations. In simulations, we consider the two cases: (i) simulations in changing the mortality $\mu$ of the infective under the fixed strength $\beta$ of the Allee effect and (ii) simulations in changing the strength $\beta$ of the Allee effect under the fixed mortality $\mu$ of the infective.

Letting the spatial region $G$ be one-dimensional region $G=(0,500)$ and setting parameters as $d_{u}=d_{v}=$ $1.0, b=1.0, \alpha=2.0, K=0.01$, under the initial distributions of the susceptible and the infective below, simulations are performed.

$$
u(0, x)=\left\{\begin{array}{cc}
1.0, & (0<x \leq 100) \\
0, & (100<x<500)
\end{array}\right.
$$

and

$$
v(0, x)=\left\{\begin{array}{cc}
0.2, & (0<x \leq 20) \\
0, & (20<x<500) .
\end{array}\right.
$$

The initial distributions in Eqs. (11) and (12) are shown in Fig. 2. As shown in Fig. 2, we consider the situation which the infective invades from the region $(0,20)$ to the region $(0,100)$ that the susceptible initially distributes in its equilibrium state $u(0, x)=1.0$ It is known that in the case where the mortality $\mu$ of the infective is rather small or large, spatially continuous or solitary traveling wave appear, however, in such cases, generated spatio-temporal patterns of the infective are comparatively simple (see Fig. 15 in Conclusions). Then, in this paper, simulations are performed in the range of the parameters that spatially complicated traveling waves appear and it is shown that such a range of parameters are very narrow.

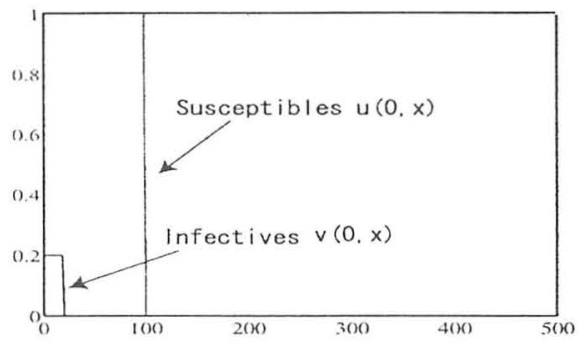

Fig. 2: Initial distributions $u(0, x), v(0, x)$ of susceptible and the infective.

I. Simulations under the Intensity $\beta=0.2$ of the Allee Effect: Changing the value of the mortality $\mu$ of the infective, spatio-temporal behaviors of the susceptible and the infective, and the influence of the random fluctuation in the proliferation rate of the susceptible on the pattern formation are studied by simulations.

(I-1) $\mu=0.445$ : Time evolution of densities of the susceptible and the infective under $\mu=0.445$ are shown in Fig. 3. The upper and the lower figures of each figure in Fig. 3 are results under the no noise and the noise.

In order to study the spatio-temporal behavior of the infective, the change in $(t, x)$-plane of the density of the infective is described in Fig. 4. (The spatiotemporal patterns of the susceptible is similar to one of the infective, so the result for the susceptible is omitted here.) We can see that the fractal structure like the Sierpinski gasket is generated in both of the no noise and noise cases.

Since it is difficult to obtain theoretically the invasion speed the infective in Fig. 4, we define the invasion speed as the gradient of the lines $A B$ and $B C$ in Fig. 4. The invasion speeds $v_{b}$ and $v_{a}$ before and after generation of the fractal structure are $v_{b} \cong 1.40$ and $v_{a} \cong$ 0.30 independently of the existence of the random noise.

(I-2) $\mu=0.435$ : Time evolution of density of the infective under $\mu=0.435$ are shown 

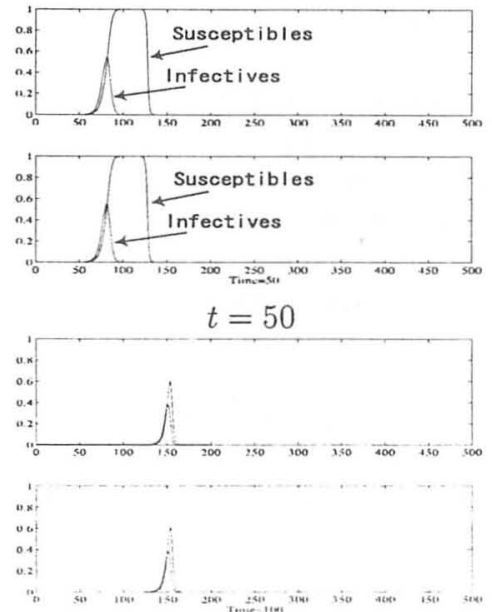

$t=100$
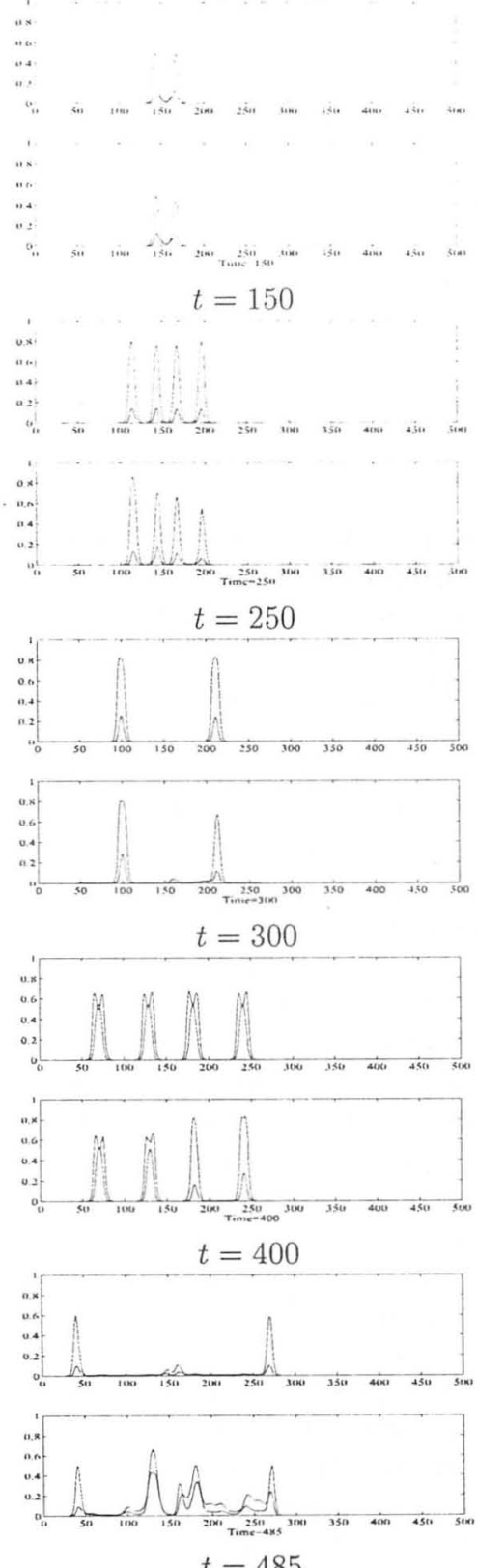

$t=485$ in Fig. 5. Figure 5 shows that the spatio-temporal behavior of the infective in the no noise case is different from one in the noise case. In the no noise case, the infective becomes extinct, however, in the noise case, some infectives go partially extinct and some infectives survive. The Allee effect causes such an extinction and on the edge of the extinction, the random noise plays an important role in survival of the infective. The invasion speeds $v_{b}$ and $v_{a}$ defined by the gradient of lines $A B$ and $B C(B C$ is parallel to $D E)$ in Fig. 5 before and after generation of the fractal structure are $v_{b} \cong 1.41$ and $v_{a} \cong 0.29$ independently of the existence of the random noise.

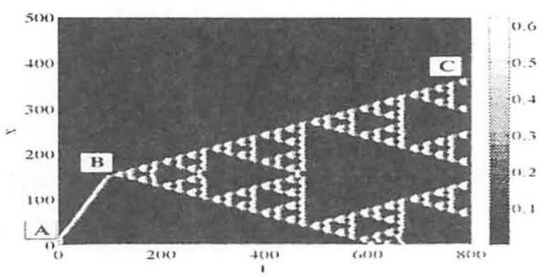

(I-1a) under the no noise

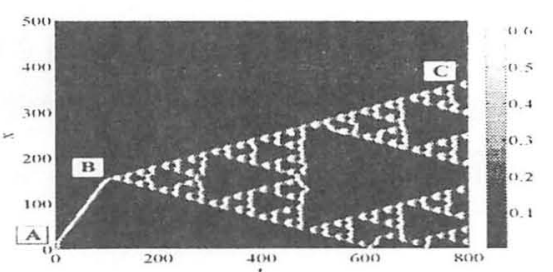

(I-1b) under the noise

Fig. 4: Spatio temporal patterns of density $v(t, x)$ of the infective with $\mu=0.445, \beta=0.2$

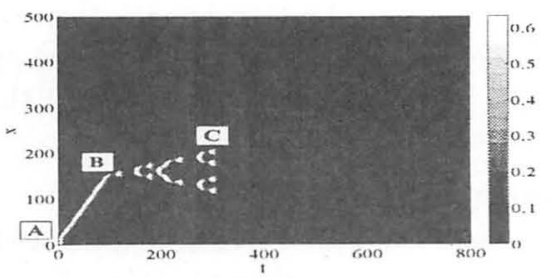

(I-2a) under the no noise

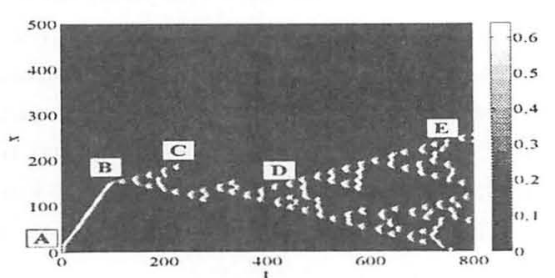

(I-2b) under the noise

Fig. 5: Spatio-temporal patterns of density $v(t, x)$ of infectives with $\mu=0.435, \beta=0.2$

(I-3) $\mu=0.432$ : Time evolution of density of the infective under $\mu=0.432$ are shown in Fig. 6. Since the mortality of the infective is less than $(I-2)$, the

Fig. 3: Snapshots of the susceptible and the infective distributions under $\mu=0.445, \beta=0.2$. 
density of the susceptible decreases larger than (I-2). Therefore, by the Allee effect, the extinction occurs in the no noise and the noise cases. The invasion speeds of the infective are the almost the same as in (I-1) and $(\mathrm{I}-2)$.

(I-4) $\mu=0.430$ : Time evolution of density of the infective under $\mu=0.430$ are shown in Fig. 7. In this case, the influence of the death rate of the infective and the Allee effect become dominant, so that the extinction occurs earlier than the case (I-3). The invasion speed of the infective is almost the same as (I-1) to (I-3) independently of the existence of the random noise.

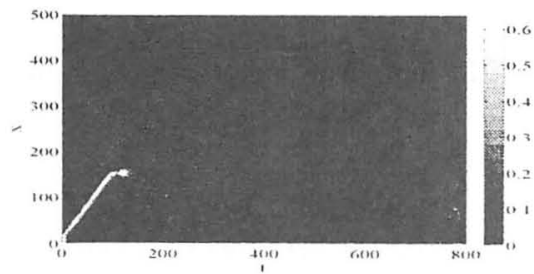

(I-3a) under the no noise

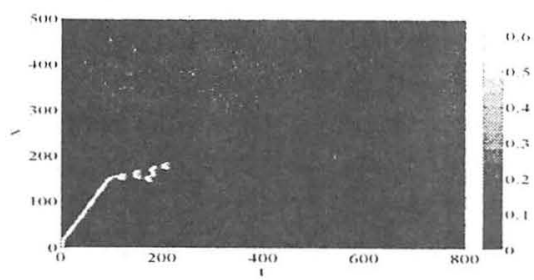

(I-3b) under the noise

Fig. 6: Spatio-temporal patterns of density $v(t, x)$ of infective with $\mu=0.432, \beta=0.2$

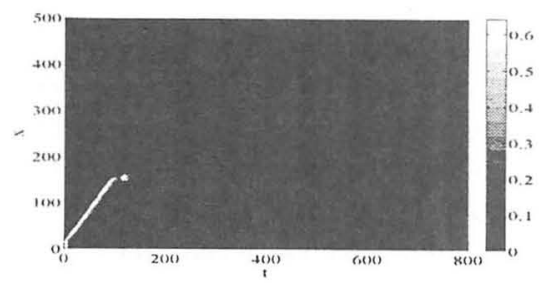

(I-4a) under the no noise

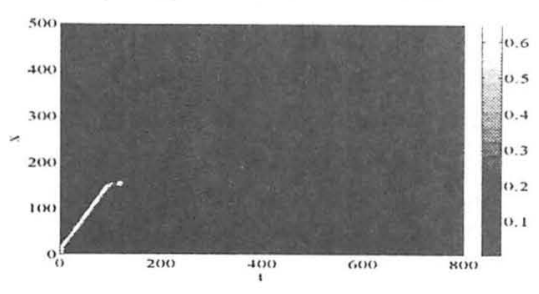

(I-4b) under the noise

Fig. 7: Spatio-temporal patterns of density $v(t, x)$ of the infective with $\mu=0.430, \beta=0.2$
II. Simulations under the Mortality $\mu=0.440$ of the Infective: In this section, changing the intensity of the Allee effect $\beta$ under the fixed mortality $\mu$ of the infective, simulations are performed.

(II-1) $\beta=0.180$ : Figure 8 shows the spatiotemporal patterns of the infective density in the case of $\beta=0.180$, the fractal structure like the Sierpinski gasket appears in both of the no noise and the noise cases. The invasion speeds $v_{b}$ and $v_{a}$ before and after generation of the fractal structure are $v_{b} \cong 1.41$ and $v_{a} \cong 0.36$ independently of the existence of the random noise.

(II-2) $\beta=0.201$ : Time evolution of densities of the susceptible and the infective under $\beta=0.201$ are shown in Fig. 9. The invasion speeds $v_{b}$ and $v_{a}$ before and after generation of the spatio-temporal structure becones $v_{b} \cong 1.41$ and $v_{a} \cong 0.300$ independently of the existence of the random noise. The invasion speed of the after the generation of the spatio-temporal structure is less than (II-1). In the noise case, the spatio-temporal structure is disturbed by the random noise. so that the regions with the high density of the infective increase.

(II-3) $\beta=0.202$ : In the no noise case, we cann see from Fig. 10 that not the fractal structure but the some congruent triangles are generated. On the other hand. in the noise case, the spatio-temporal structure is disturbed and the propagation of the disease is broken off in some regions. The invasion speeds $v_{b}$ and $v_{a}$ before and after generation of the spatio-temporal structure becomes $v_{b} \cong 1.41$ and $v_{a} \cong 0.295$ independently of the existence of the random noise. The invasion speed of the after the generation of the spatio-temporal structure is less than (II-1) and (II-2).

(II-4) $\beta=0.205$ : In this case, we can see from Fig. 11 that the extinction of the infective occurs because of the strong intensity of the Allee affect.
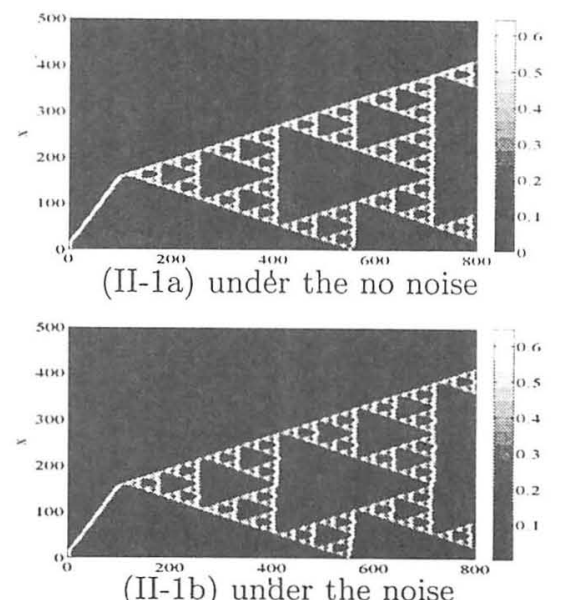

Fig. 8: Spatio-temporal patterns of the infective density $v(t, x)$ under $\mu=0.440, \beta=0.180$ 


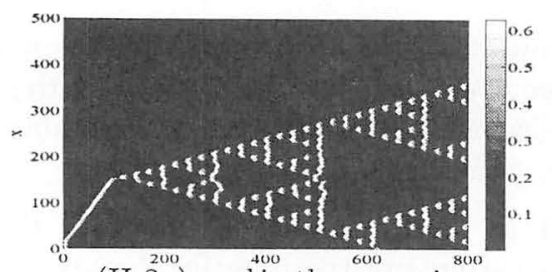

(II-2a) undèr the no noise

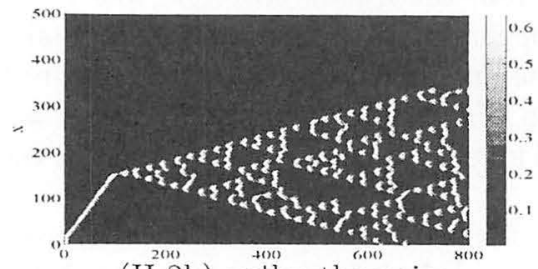

(II-2b) under the noise

Fig. 9: Spatio-temporal patterns of the infective density $v(t, x)$ under $\mu=0.440, \beta=0.201$

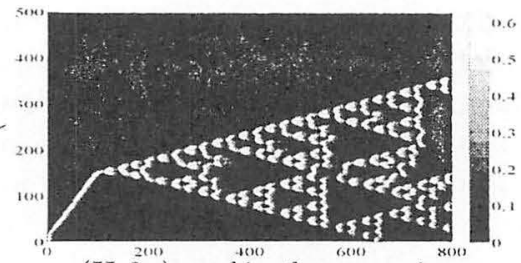

(II-3a) under the no noise

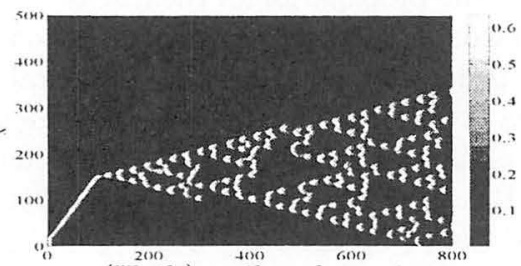

(II-3b) under the noise

Fig. 10: Spatio-temporal patterns of the infective density $v(t, x)$ under $\mu=0.440, \beta=0.202$

Comparing results of simulations in $(\mathrm{II}-1) \sim(\mathrm{II}-4)$, the propagation speed of the diseases seems to be inversely proportional to the intensity of the Allee effect.

Although the simulation results are omitted here, under the intensity $\beta=0.207$ of the Allee effect and the death rate $\mu=0.440$ of the infective, the Allee effect becomes to dominate than the random noise in the proliferation, so that the susceptible and the infective extinct in the both of the no noise and noise cases.

\section{Conclusions}

We have studied the influence of the Allee effect, the random fluctuation in the proliferation of the susceptible and the mortality of the infective on the spatiotemporal behaviors in the stochastic diffusive SI system by the numerical simulations. From the results of simulations, we have obtained the following results:

(i) The propagation speed of the diseases is inversely

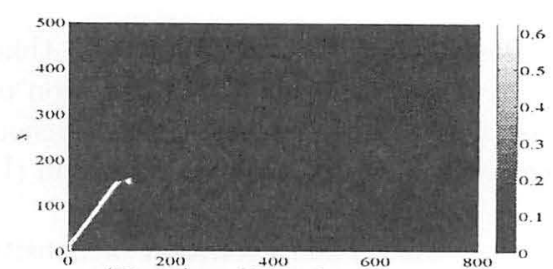

(II-4a) undèr the no noise

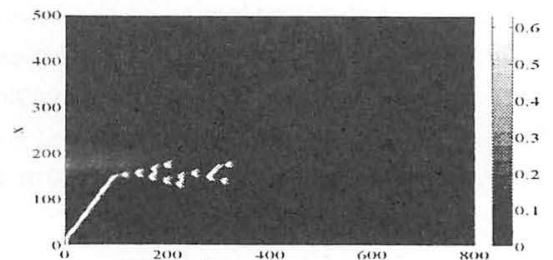

(II-4b) under the noise

Fig. 11: Spatio-temporal patterns of the infective density $v(t, x)$ under $\mu=0.440, \beta=0.205$

proportional to the intensity $\beta$ of the Allee effect.

(ii) The influence of the random fluctuation in the proliferation of the susceptible on the spatio-temporal behaviors of the infective( susceptible) is proportional to the intensity $\beta$ of the Allee effect.

(iii) By the interaction of the Allee effect, the mortality of the infective and diffusion, the situations below appear:

(iii-a) In some cases, the spatio-temporal patterns of the infective(susceptible) generate the fractal structure like the Sierpinski gasket. The fractal structure is maintained under the random noise if the intensity $K$ of the random noise is less than 0.2 . The generation of the fractal structure implies that the region with the infective has regularity, so in this case, we can estimate the region with the infective.

(iii-b) Depending on existence or nonexistence of the random fluctuation in the proliferation of the susceptible, epidemic processes of the diseases drastically change in some cases. Even if the extinction of the infective(susceptible) occurs in the case of the no noise, the diseases persist in some regions under the noise.

Spatio-temporal patterns in the first quadrant of $(\mu, \beta)$-plane under the no noise and the noise are shown in Fig. 12. The straight line on the bottom-right corner in Fig. 12 shows the Hopf bifurcation line. On the right side region of the Hopf bifurcation line, the uniform propagation (Fig. 13) of the disease occurs in the no noise and the noise cases. In the upper region of the symbol " $\triangle$ " are the region that the extinction of the susceptible and the infective occurs, the regions with " $x "$ and " $\bigcirc "$ are the regions that the solitary traveling wave as shown in Figs. 14 and 15 and the fractal structure like the Sierpinski gasket are generated. From Figs. 13 and 14, we can see that the spatio-temporal behaviors of the infective are sensitive to the parameter and the existence of the random noise. It follows from 
Figs. 12 to 15 that the range of parameters $\beta$ and $\mu$ that the solitary wave or the Sierpinsky gasket is generated is narrow. Such ranges in the noise case is smaller than the no noise case.

The Sierpinski gasket in the reaction-diffusion equation was firstly founded in [5], [6], however, the models in [5], [6] are essentially different from the model proposed here in that our model is stochastic and has different kinetics.

This work is supported by The Ministry of Education, Culture, Sports, Science and Technology under Grantin-Aid for Research (C) 19560444 .

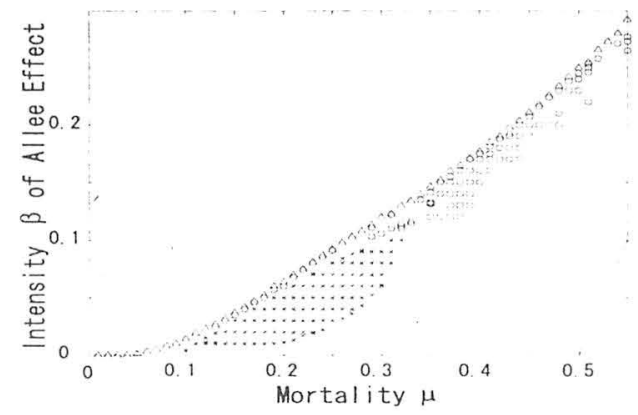

(a) under the no noise

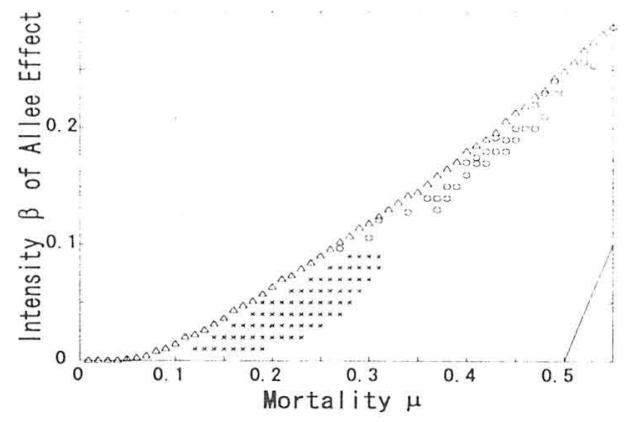

(b) under the noise

Fig. 12: Classification of spatio-temporal patterns in $(\beta, \mu)$-plane. $\times$ :solitary wave, o:the Sierpinski gasket, $\triangle$ :lower limit of the extinction
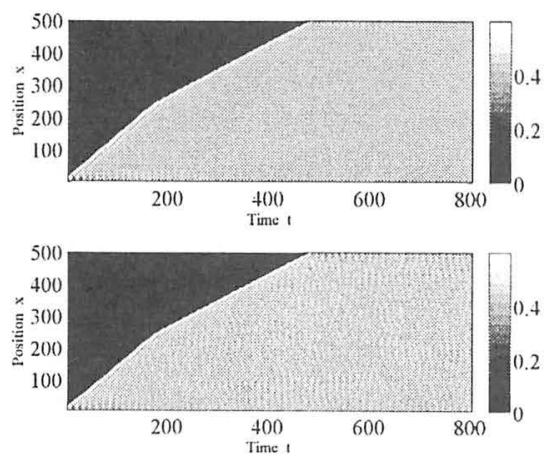

Fig. 13: Uniform propagation of the the infective under $\beta=0.06, \mu=0.54$ (upper: the no noise case, lower: the noise case)
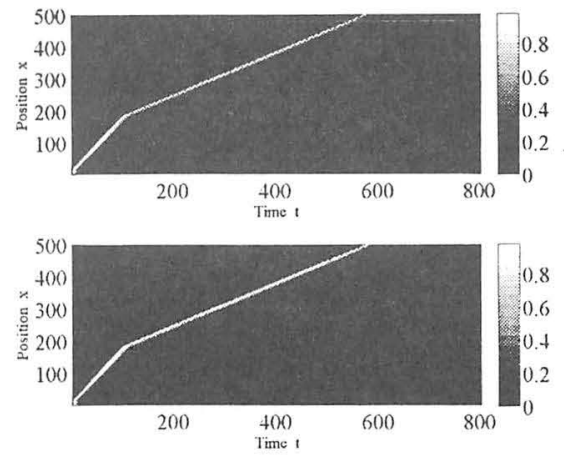

Fig. 14: Solitary wave of the infective under $\beta=0.06$, $\mu=0.29$ (upper: the no noise case, lower: the noise case)
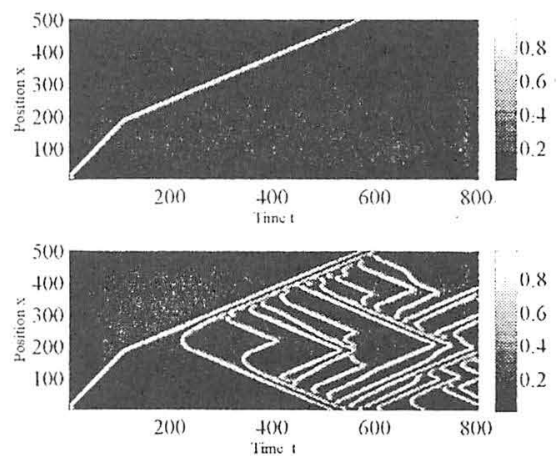

Fig. 15: Solitary wave of the infective under $\beta=0.06$, $\mu=0.30$ (upper: the no noise case, lower: the noise case)

\section{References}

[1] J. D. Murray, Mathematical Biology I, II, Springer, 2003.

[2] Anderson H. and Britton T. "Stochastic Epidemic Models and Their Statistical Analysis, "Springer, 2000.

[3] Petrovskii S. V. H., Morozov A. and Li B-L., "Regimes of Biological Invasion in a Predator-prey System with the Allee Effect," Bulletin of Mathematical Biology, 67, 637/661, 2005.

[4] Petrovskii S. V., Morozov A. Y. et al., "Allee Effect makes Possible Patchy Invasion in a Predator-prey System," Ecology Letters," 5, 345/352, 2002.

[5] Y. Hayase, T. Ohta, "Self-replicating Pulses and Sierpinski Gasket in Excitable Media," Physical Review E, 62, 5, 5998/6003, 2000.

[6] Y. Hayase, T. Ohta, "Sierpinski Gasket in a Reaction-Diffusion System," Physical Review Letters, 81, 8, 1736/1729, 1998. 